\title{
Memorias de un servicio de cirugía general y de sus cirujanos. Parte 1
}

\author{
Memories of a general surgery service and its surgeons. Part 1
}

\author{
David Olvera Pérez*
}

\section{Palabras clave: Cirugía general, cronología, cirujanos, seguridad social. \\ Keywords: General surgery, timeline, surgeon, security health.}

* Asociado Titular de la Asociación Mexicana de Cirugía General.

Recibido: 27/02/2019 Aceptado: 07/08/2019

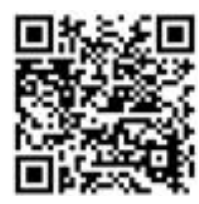

\section{RESUMEN}

Esta es una cronología del Servicio de Cirugía General y de los cirujanos generales del Hospital General del Centro Médico Nacional del IMSS (1963-1981), hospital que desapareció con el sismo de 1985 y que, sin duda alguna, formó parte importante de la historia de la Cirugía General en México. En estos artículos honramos y homenajeamos a nuestros maestros que nos forjaron y dieron vida profesional. Tengo la esperanza que muchos cirujanos envíen a nuestra revista memorias semejantes para enriquecer nuestro acervo cultural de Cirujanos y Cirugía General en México.

\section{ABSTRACT}

Timeline of the General Surgery Service and the general surgeons of the General Hospital of the National Medical Center of the Mexican Social Security Institute 1963-1981 (HG of the CMN of the IMSS). That disappeared with the 1985 Earthquake, without a doubt it was an important part of the History of General Surgery in Mexico. In these articles we honor and honor our teachers who forged and gave us professional life. I hope that many surgeons will send similar memories to our magazine to enrich our cultural heritage of Surgeons and General Surgery in Mexico.
La historia, émula del tiempo, depósito de las acciones, testigo de lo pasado, ejemplo y aviso de lo presente, advertencia de lo porvenir. Miguel de Cervantes

$E^{\prime}$ maestro Manuel Quijano Narezo y la mayoría de los cirujanos notables y eminentes del Servicio original de Cirugía General han muerto. Indiscutiblemente, merecen ser recordados y homenajeados por su labor personal y de "equipo", la cual forjó una parte importante de la cirugía general de México.

\section{PRESENTACIÓN}

En esta descripción histórica - se podría decir, novelesca-del Servicio de Cirugía General y de los cirujanos que participaron en los inicios del Hospital General del Centro Médico Nacional del Instituto Mexicano del Seguro Social (HG del
CMN del IMSS), me he permitido recordar una parte importante de mi formación profesional, que con gusto comparto con todos los cirujanos generales y que, por supuesto, volvería a vivir. Porque, como dice la frase, "recordar es vivir".

Las emociones se juntan cuando se trata de escribir los recuerdos con la mayor veracidad; casi siempre existe el riesgo de omitir algo, pues la memoria falla. Por lo mismo, ofrezco disculpas por las omisiones involuntarias y por la falta de información que me impide ser más certero en esta descripción.

En fechas recientes, después del fallecimiento de algunos cirujanos que más adelante describiremos, estuve presente en eventos académicos en los que faltaron palabras póstumas bien merecidas para ellos. Los líderes en ese momento no consideraron realizarlas, seguramente por no ser de su conocimiento histórico el valor que tuvieron sus actos y lo que hicieron para mejorar la cirugía general en México, eso 
que nos llevó a ser lo que somos actualmente. La historia en la guerra y en la política la escriben los triunfadores, pero, en la vida real, solamente la saben los que la presencian; ésta es una de ellas.

Henry Sigerist insistía en que la historia de la medicina puede ser un útil mediador entre el antiguo humanismo y la ciencia moderna; una idea que parece casi olvidada en nuestros días. Durante esta expuesta en Leipzig, Sigerist colocó a la historia de la medicina dentro de un marco con un mayor contexto cultural, y se pronunció por un programa de investigación. ${ }^{1}$

Para saber qué pasó y por qué pasó, debo remontarme a los orígenes de los hechos, los cuales, indiscutiblemente, sufren cambios por el hombre, por la necesidad de mejorar las circunstancias. Además, de acuerdo con el historiador Enrique Flores Cano, es una forma de enseñar la diversidad del ser humano y de su creatividad en su desarrollo social. ${ }^{2}$

El objetivo de esta relato es, describir, pero sobre todo, realizar un homenaje a los médicos y cirujanos que nos forjaron. Como cirujano del Servicio de Cirugía General del Hospital General del CMN del IMSS que participé durante esos años, es muy interesante rememorar la evolución histórica de un servicio de cirugía general que, en su tiempo, fue líder en asistencia y producción académica para congresos, vanguardista en muchas patologías quirúrgicas y en formación de cirujanos. El compartir estos hechos históricos de la cirugía general, creo, nos permitirá conocernos mejor, entender nuestro pasado quirúrgico y prepararnos mejor para el futuro.

Mi paso por el HG del CMN del IMSS fue relativamente corto, tan sólo cuatro años de residente y siete años de cirujano. Aunque, sin duda, lo más importante fue estar allí en los mejores años de productividad, desarrollo, investigación académica y reconocimientos.

Una vez más, por medio de este relato, agradezco a todos y a cada uno de los que participaron en mi formación profesional, porque estoy en deuda con ellos. Durante mi estancia, recibí una preparación como muchos compañeros residentes, pero muy pocos tuvimos la oportunidad de colaborar profesionalmente en ese centro médico asistencial, de avanzada, y de recibir elogios —en mi caso, inmerecidos - por su trabajo arduo durante los primeros 10 años.
Cicerón dijo que "la historia es testimonio del tiempo, luz de la verdad, vida de la memoria, maestra de la vida, reflejo de la antigüedad". ${ }^{3} \mathrm{~A}$ través de estas líneas, invito a todos los cirujanos generales de México que envíen reseñas y semblanzas de sus maestros, para que los anales de la Asociación Mexicana de Cirugía General se enriquezcan con esta información y, al mismo tiempo, para que las nuevas generaciones entiendan y conozcan mejor la evolución de la cirugía general en México.

Para mejorar la calidad del tema y su descripción, invite al Dr. Rafael Álvarez Cordero, un cirujano brillante y un escritor sobresaliente, quien vivió y participó activamente con su trabajo profesional en estos relatos. Quiero aprovechar este espacio para agradecerle su tiempo.

\section{ORIGEN}

El 15 de octubre de 1943, el presidente de la República Mexicana, el general Manuel Ávila Camacho, dictó un acuerdo para unificar los Servicios de Salubridad y Asistencia Pública. En ese entonces, el secretario en funciones era el Dr. Gustavo Baz Prada, quien asumió esa gran responsabilidad; de esta manera, con el objetivo de evitar la duplicidad y el gasto innecesario, buscó una mayor coordinación para lograr la eficacia suficiente y el incremento de la cobertura hospitalaria en toda la República mexicana. De eso, lo relataré más adelante.

Por esas fechas, se comentaba que cuidar la salud del pueblo mexicano era cuidar la de la Patria misma, ya que solamente mejorando la salud de quien labora se podría construir una Patria sana y fuerte. Hasta hoy, esas palabras siguen vigentes.

La Secretaría de Salubridad y Asistencia inició un estudio para formular y ejecutar un proyecto de construcción de instituciones hospitalarias, cuyo objetivo era mejorar sustancialmente los servicios de atención médica para la población, considerando que los viejos hospitales eran poco adaptables para funcionar conforme a los avances modernos de la medicina de ese tiempo. Así, más de 200 hospitales, entre grandes y chicos, construidos a lo largo y ancho de nuestro país, conformaron la cifra que ha quedado para la historia en materia de construcción por una sola administración de salud. ${ }^{4}$ 
Fuera de contexto universal, indudablemente, esa generación de políticos y administradores de la salud fueron grandes personajes. Su pensamiento fue más allá de lo ordinario; querían que las instituciones con el tiempo, como el Seguro Social, fueran creciendo y desarrollándose. Sólo así los cálculos de la capacidad de la Secretaría de Salubridad de ese entorno podrían considerarse desde otro punto de vista, pero, cuando eso sucediera, los hospitales emprendidos estarían al nivel de calidad de cualquier hospital que levantaran otras instituciones y organismos. Y así sucedió en el campo de la asistencia.

Pero volviendo a la década de los cuarenta, la red hospitalaria planeada por la Secretaría de Salubridad y Asistencia tenía contemplada una construcción especial para la capital del país, un centro médico (impensable para su tiempo) grande y completo, que fuera superior a muchos existentes y en igualdad a otros. Siendo el Dr. Baz ministro de Salubridad y Asistencia (3 de septiembre de 1945) y a un año de terminar su gestión, fue expedida la ley que creó el Comité de Proyección y Construcción del Centro Médico de México. Sus objetivos eran asistir a la población del Distrito Federal y aledaña, con los mayores adelantos de vanguardia médica de su tiempo, reducir costos y mejorar la asistencia social. Esta ley estaría conformada por 12 grandes hospitales con equipo y tecnología moderna que abarcarían todas las ramas de la medicina. Y por nombre llevaría la "Magna Ciudad de la Salud".

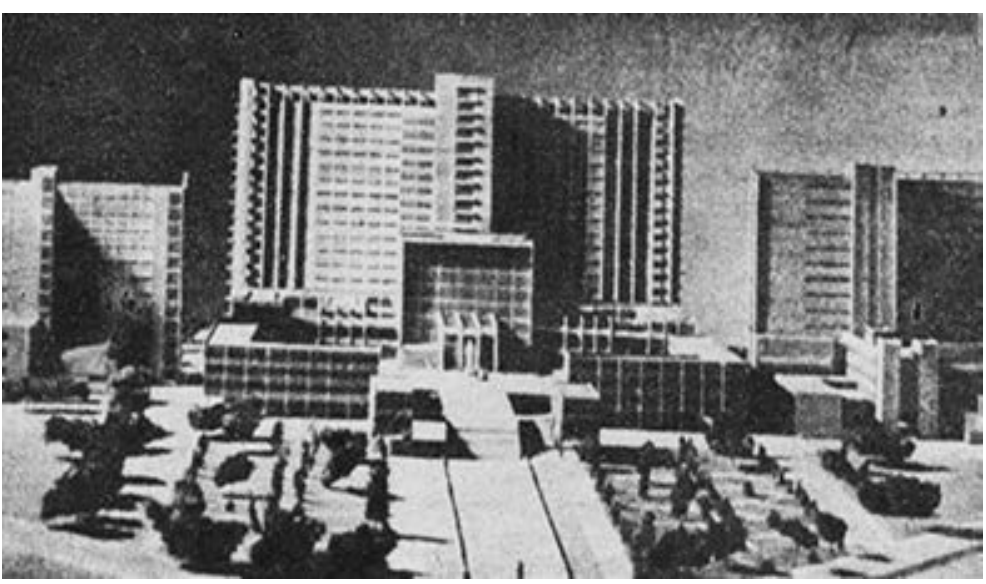

Figura 1: Maqueta de tres de los principales establecimientos de la "Magna Ciudad de la Salud".
Las Figuras 1 y 2 se remontan al año de 1945, en la primera, se trata de una maqueta de tres de los principales establecimientos del Centro Médico de la Ciudad de México. Y en la segunda, es una composición en la que puede observarse la glorieta de la Av. Cuauhtémoc (que no se si existió), y lo que serían diversos hospitales. En esa fecha, estaban terminados el Hospital del Niño y el Instituto de Cardiología y tres hospitales más se habían empezado a construir.

El Centro Médico Nacional Siglo XXI del Instituto Mexicano del Seguro Social tiene una larga historia, la cual se remonta a la primera mitad del siglo XIX. Dentro de sus múltiples hospitales, y al interior de los muros de esos hospitales, seguramente, existe una gran variedad de historias médicas y de pacientes, incluso de los mismos hospitales. A lo largo de estas memorias, relataremos el origen y el fin de un servicio de cirugía general que existió en el Hospital General del Centro Médico Nacional del Instituto Mexicano del Seguro Social.

\section{INICIO Y EVOLUCIÓN}

El 15 de marzo de 1963, el presidente de México, el Lic. Adolfo López Mateos, inauguró el Centro Médico Nacional del Instituto Mexicano del Seguro Social (IMSS). En dicha inauguración mencionó solemnemente que el IMSS cumplía su vigésimo aniversario y que sería memorable por llevar la salud y el bienestar a los trabajadores del país (Figura 3). ${ }^{5}$

El primero de diciembre de 1958, el Lic. Benito Coquet había sido designado como director del Instituto Mexicano del Seguro Social por designación del presidente Adolfo López Mateos. Durante la primera quincena de su gestión, presentó para consideración del presidente de la República los nombres de las personas que propondría al Consejo técnico del instituto. De esta manera, se nombró como Subdirector General Médico al Dr. Luis Méndez; asimismo, el Dr. Bernardo Sepúlveda aceptó la Jefatura del Departamento de Planeación y Organización de los Servicios Médicos.

El Lic. Benito Coquet escribió en ese entonces:

El inicio de las actividades del Instituto Mexicano del Seguro Social, casi simultánea- 


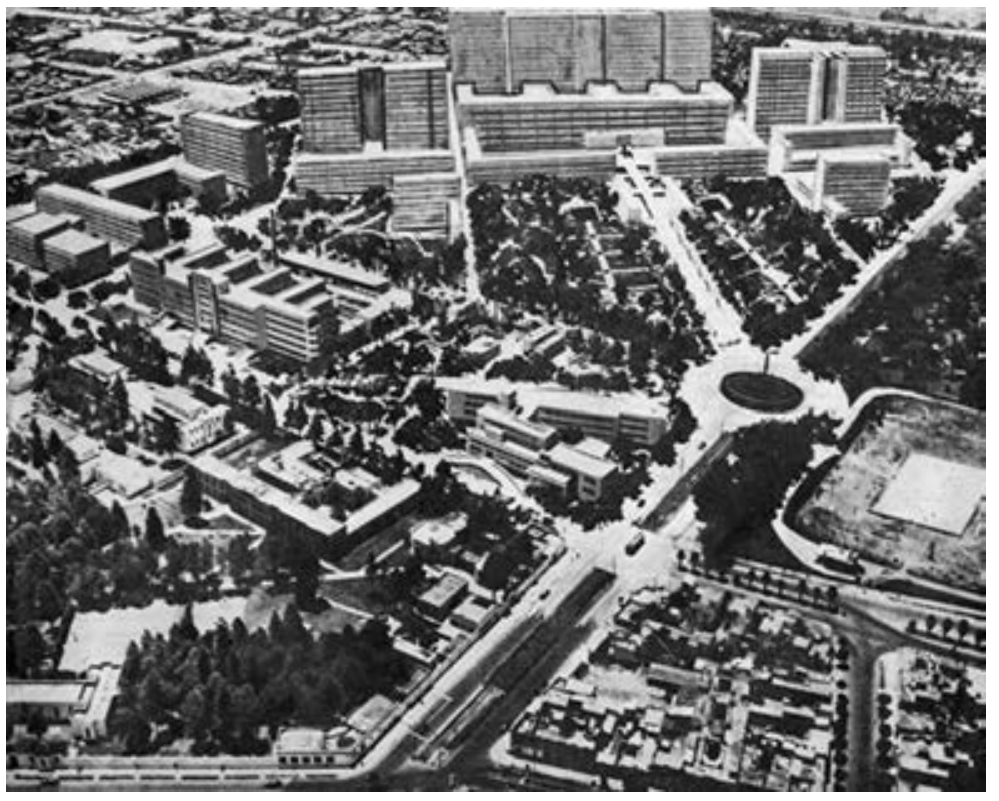

Figura 2: En esta composición fotográfica, se puede observar que el Hospital del Niño y el Instituto de Cardiología ya estaban terminados, y lo que serían varias instituciones médicas que conformarían la "Magna Ciudad de la Salud".

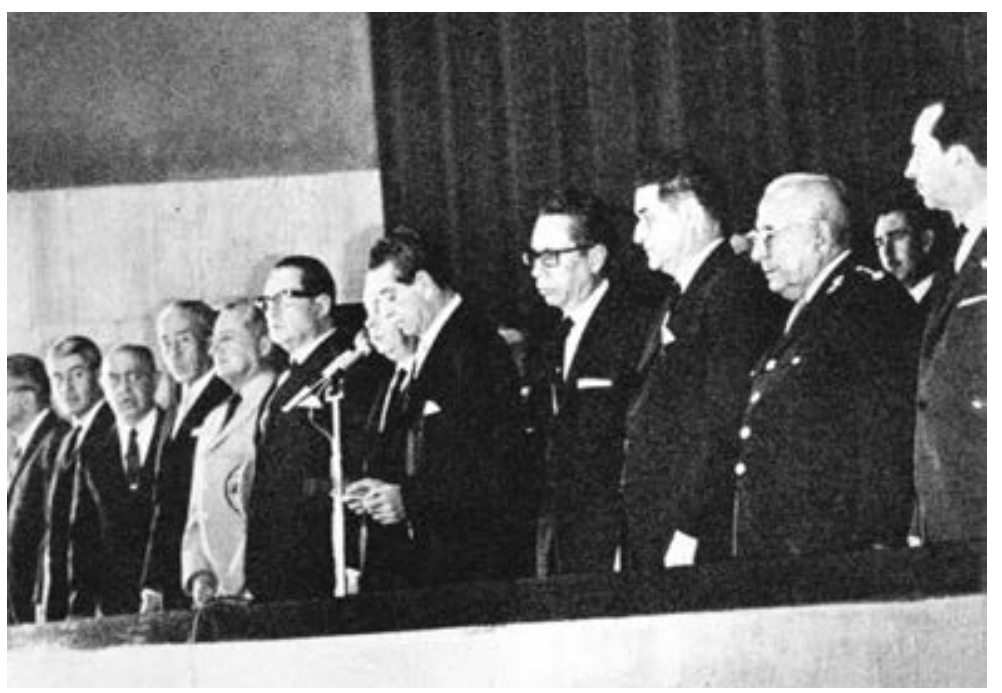

Figura 3: Inauguración del Centro Médico Nacional por el Lic. Adolfo López Mateos, presidente de México.

mente al de la planeación e inicio de la construcción del Centro Médico, pareció vincular, desde sus origines, a uno y a otro, no obstante, el Seguro Social nacía como un organismo descentralizado del estado, y el Centro Médico se proyectaba como una dependencia de la en- tonces recién creada Secretaría de Salubridad y Asistencia. Casi 19 años después de haberse iniciado la construcción del Centro Médico, el Seguro Social adquirió por enajenación lo edificado hasta entonces y dio por término la construcción de las distintas unidades que lo integraban; la última, el Hospital MédicoQuirúrgico (que finalmente fue identificado como el Hospital General del Centro Médico Nacional del Instituto Mexicano del Seguro Social), fue terminado y puesto en servicio el 15 de marzo de 1963.

Hasta aquí las palabras del Lic. B. Coquet [sic].

Y como menciona el Lic. B. Coquet, así sucedió. La historia señalará lo que el Centro Médico significó para la población trabajadora y para el avance de la medicina. Pues, por casi 25 años, fue una institución de la que el país pudo enorgullecerse: cumplió una tarea para el bien de México y de los mexicanos. Además, indudablemente, fue hermoso desde el punto de vista arquitectónico; un conjunto hospitalario que sus atributos laborales, asistenciales, y académicos, ganados durante su vigencia, le permitieron presumir de ser el más importante de México y, probablemente, de América Latina.

En el libro conmemorativo de la inauguración, en el primer capítulo dedicado al Hospital General, Ilama la atención que en la lista de especialidades médicas instaladas en esa unidad no aparezca el Servicio de Cirugía General. No obstante, esta ausencia tiene una explicación. La cirugía general se consideraba en México como un apéndice grande de la gastroenterología (desconozco el origen de esta condición que no tenía razón de ser, pues, por ejemplo, los servicios quirúrgicos en los países europeos, desde siempre se han considerados independientes). El maestro Bernardo Sepúlveda, ya como jefe del Departamento de Planeación y Organización de los Servicios Médicos del IMSS, colocó al Dr. Luis Landa Verdugo como Jefe del Servicio de Gastroenterología del HG del CMN del IMSS. Los dos venían del Hospital de Enfermedades de la Nutrición, que hoy se conoce como Instituto Nacional de Ciencias Médicas y Nutrición "Salvador Zubirán", en donde el Servicio de Cirugía General estaba adscrito al Servicio de Gastroenterología, por lo que adoptaron e ins- 
talaron ese modelo de asistencia y enseñanza, el cual persiste a la fecha.

El Hospital General del CMN del IMSS inició sus actividades ese mismo mes de marzo de su inauguración (1963) (Figuras 4 y 5). Así, el Dr. Gustavo Baz Díaz Lombardo fue el pri-

I NSTITUTO MENICANO

DEL S E G U R O SOC I A L

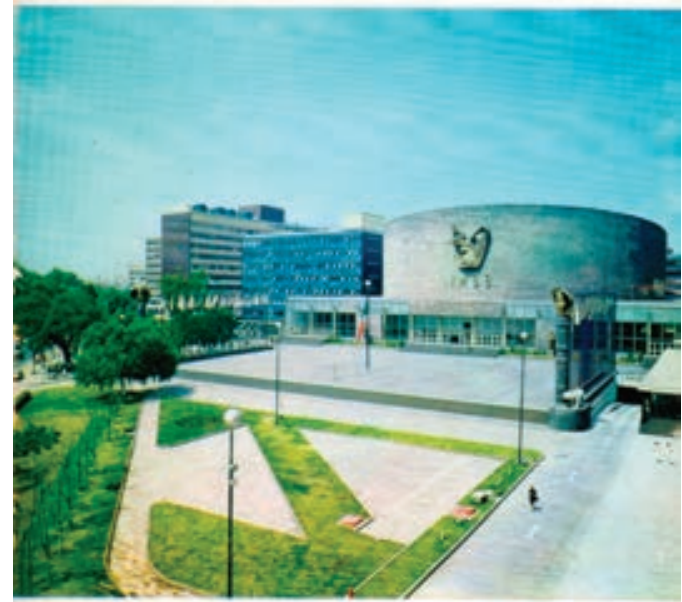

\section{CE N T R O MEDICO} N A C I O N A L

MEXICO, D. F.

Figura 4: Explanada principal del CMN.

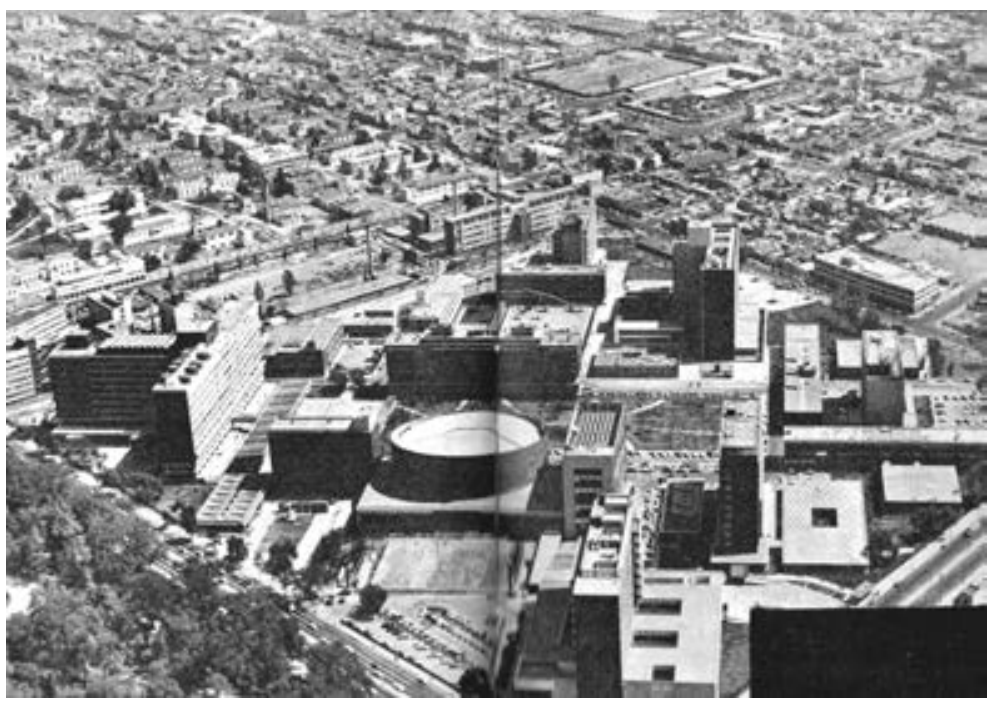

Figura 5: Panorámica final del CMN del IMSS. mer Cirujano General que se presentó e inició inmediatamente las actividades quirúrgicas. El Dr. Francisco Hidalgo Castro llegó como Residente de Cirugía, ya con entrenamiento quirúrgico (zincompleto?) del Hospital de Enfermedades de la Nutrición. El Dr. Jorge Bautista O'Farrill se integró en el mes de mayo, y tres meses después de la llegada del Dr. Hidalgo, el Dr. Rafael Álvarez Cordero se agregó como el segundo residente. Y el Dr. Vicente Guarner Dalias se integró al equipo en el mes de agosto del mismo año.

De acuerdo con el Dr. Félix Ramírez Espinosa, un excelente Exresidente del Servicio de Angiología y amante de sus orígenes de especialidad, mencionó que la residencia de Cirugía General fue un pilar importante en el desarrollo del Hospital General; ésta fue la primera residencia en ese hospital, y por lo mismo, prestó apoyo médico a todas las especialidades que la requerían. ${ }^{6}$

Los primeros meses fueron de gran intensidad quirúrgica. Como se ha relatado, el Dr. Baz prácticamente vivía en el hospital, pues operaba tanto en el turno matutino, vespertino y nocturno; así nació la leyenda que en una guardia de fin de semana operó a 38 pacientes, récord que nunca fue superado por las generaciones subsecuentes, incluyéndome (con un límite de 28 cirugías y que, a diferencia, yo tenía un mayor apoyo con más residentes). Los primeros equipos de cirugía quedaron integrados, por un lado, por el Dr. Gustavo Baz Díaz Lombardo y el Dr. Francisco Hidalgo y, por el otro, por el Dr. Jorge Bautista O'Farrill con el Dr. Rafael Álvarez Cordero.

En el siguiente año, el Dr. Francisco Hidalgo fue incorporado como cirujano de base, y llegaron a la residencia quirúrgica los Dres. Isauro Duran Llamas y Carlos Godínez Oropeza.

El Dr. Manuel Quijano Narezo fue invitado a colaborar en el IMSS por su prestigio, al ser en ese tiempo uno de los mejores cirujanos de México, quien durante los primeros dos años fungió como director, pero designado como subdirector. Aunado a su prestigio profesional, lo avalaba el haber realizado, a propuesta del Dr. Jorge Bautista O'Farril, la colecistectomía electiva a la esposa del presidente, el Lic. Gustavo Díaz Ordaz. El Dr. Rafael Álvarez 
Cordero tuvo la fortuna de haber participado en esa cirugía.

El Dr. Quijano se formó como cirujano en el Hospital General de México y fue cirujano del Hospital de Enfermedades de la Nutrición, quien llegó a la Dirección del Hospital General del CMN del IMSS, y tomó la Jefatura de Cirugía General, pero aceptó el sistema médicoquirúrgico con la coordinación del Servicio de Gastroenterología. En esta etapa se fundan las actividades académicas y asistenciales.

Cuando el Dr. Luis Landa inició la jefatura con férrea disciplina, trasmitió su responsabilidad en la asistencia, dedicación a la academia e invitación a la investigación. El Servicio de Gastroenterología en su inicio ocupaba todo el séptimo piso del Hospital General, el cual tenía una conformación arquitectónica en T, por lo que el ala norte era ocupada por pacientes masculinos y el ala sur por pacientes femeninos; en el segmento oriente-poniente se encontraban los Servicios de Consulta Externa, Laboratorio Clínico de Investigación, de Bioquímica, Bacteriología y Amibiasis. El Servicio de Endoscopia en ese entonces era manejado por el Dr. José Ramírez Degollado, gran pionero en este ramo. Para el año de 1964, debido a la gran demanda de pacientes gastroenterológicos, el área de hospitalización se duplicó hasta integrarse el sexto piso, llegando a las 100 camas.

De acuerdo con esta distribución de pacientes, existía un gastroenterólogo y un cirujano por cada sección, de tal forma que el Dr. Alfonso Perches y el Dr. Jorge Bautista formaban un solo equipo; el Dr. Antonio de León y el Dr. Gustavo Baz Díaz Lombardo formaban otra pareja, así como también los doctores Luis F. Cervantes y Vicente Guarner, y los doctores Enrique Segovia y Francisco Hidalgo. Y la rotación se realizaba cada año. Para cada una de estas secciones, se agregaban los residentes de 2o., 3er. y 4o. año de Cirugía General, además de los Residentes de Gastroenterología Médica.

La organización continuó y, para el año de 1967, cuando el Dr. Carlos Godínez terminó su residencia, fue aceptado como médico de base. En ese tiempo ya existían cuatro cirujanos en el turno matutino, y con él se completó el turno vespertino y nocturno que iniciaba a las 4 p. m., y terminaba a las 8 a. m. del día siguiente; los doctores Rafael Álvarez Corde- ro, Carlos Godínez Oropeza y Raúl Gómez Garza fueron los pioneros de este servicio. Así funcionó hasta el año de 1972, cuando el Dr. Gustavo Baz Díaz Lombardo renunció para fundar e iniciar el Sistema de Salud de Ciudad Nezahualcóyotl del Estado de México. El Dr. Carlos Godínez ocupó el turno matutino, y el Dr. Luis Ize Lamache, Residente del 3er. año bis (así se denominaba el cuarto año de Cirugía General) y Jefe de Residentes, ocupó el turno de guardias. En el mismo año, el Dr. Ize fue becado para asistir al 1er. Curso de Nutrición Parenteral en Estados Unidos de Norteamérica, por lo que a su regreso se dedicó a formar el Servicio de Nutrición Parenteral y su turno de guardia nocturna la ocupó el que suscribe esta cronología). Esta distribución permaneció durante dos años.

Ya en el año de 1975, la reorganización de la estructura del HG CMN del IMSS, promovida por el Dr. Gilberto Flores Izquierdo, quien sustituyó al Maestro Manuel Quijano Narezo en la dirección del hospital, nombró al Dr. Rafael Álvarez Cordero como Jefe de la División de Cirugía, y al Dr. Jorge Bautista O’Farrill como Jefe del Servicio de Cirugía General; de esta manera, se terminaba el binomio de gastroenterología y cirugía y, así, se iniciaba otra etapa en la historia de la cirugía.

El Dr. Rodolfo Rojas Rubí cubrió la guardia y laboró por un tiempo breve en el servicio. Fue llamado por el Director General del IMSS a organizar el Servicio de Pacientes VIP (very important people, por sus siglas en inglés), en donde descubrió sus dotes administrativas y políticas que lo llevaron a desempeñar posteriormente diversos cargos administrativos en el Instituto de Seguridad y Servicios Sociales de los Trabajadores del Estado (ISSSTE) y en la Secretaría de Salubridad y Asistencia (SSA). En el mismo año, el Dr. Raúl Gómez Garza llegó para continuar la labor del Dr. Gustavo Baz en el Sistema de Salud de Ciudad Nezahualcóyotl, y también los doctores Roberto Blanco Benavides y Gustavo Ramírez Wiella se incorporaron al Servicio de Cirugía.

Al poco tiempo, continuaron los cambios; el Dr. Vicente Guarner sustituyó al Dr. Rafael Álvarez Cordero en la División de Cirugía por un tiempo breve, quien dejó ese cargo al emigrar al Centro Médico "La Raza" del IMSS 
para responsabilizarse del Servicio de Cirugía General.

Los residentes de cirugía general que tuvimos la fortuna de forjarnos en ese servicio mixto de gastro-cirugía asistíamos a las sesiones conjuntas, además de nuestras clases de cirugía. Así, participábamos de las sesiones bibliográficas, radiológicas y de patología.

El Dr. Manuel Quijano Narezo, primer Director del Hospital General del CMN, escribió: "que este Hospital fue insignia del Centro Médico Nacional y contribuyó, como ningún otro, a dar prestigio académico y profesional al Instituto Mexicano del Seguro Social". ${ }^{6}$ La participación del Servicio de Gastroenterología del HG del CMN del IMSS en los congresos nacionales de la Asociación Mexicana de Gastroenterología fue muy importante, hasta con un $40 \%$ de los trabajos libres, y en el mismo porcentaje en conferencias, simposios y mesas redondas. Para muestra, el VII Congreso Nacional de Gastroenterología realizado en el año 1971, donde se presentaron 20 trabajos libres de 60 (33\%), y en el IX Congreso Nacional de Gastroenterología del año 1975 fue el 32\%.

El Servicio de Gastroenterología (y Cirugía) tuvo vigencia de 1963 a 1975, y durante esos años, su imagen creció tanto en la República como fuera de ella, por los protocolos de patologías gastrointestinales, así como por la investigación. Queda constancia en el número importante de médicos de Centro y Sudamérica que adquirieron destrezas quirúrgicas en este servicio. Los temas gastrointestinales en los que siempre estuvo el Servicio de Gastroenterología a la vanguardia fueron: la hipertensión portal, el manejo de la enfermedad péptica, el tratamiento de la pancreatitis (en 1980, 600 pacientes documentados por el Dr. López Fournier, quien personalmente me lo dijo). Los primeros estudios de la obesidad mórbida, tratamiento de las complicaciones de la amibiasis, hepatologías y nutrición parenteral.

Los cambios en el organigrama hospitalario se produjeron también en los servicios. En el año de 1975, el Servicio de Gastrocirugía sufrió la disrupción, separándose en Cirugía General y Gastroenterología. Así nació el Servicio de Cirugía General en el Hospital General del Centro Médico Nacional del IMSS. Su primer jefe fue el Dr. Jorge Bautista O'Farrill, y se le asignó el sexto piso completo, una sección para pacientes femeninos y la otra para los masculinos. Los cirujanos que iniciaron esta transición fueron: el Dr. Carlos Godínez Oropeza, el Dr. Luis Ize Lamache, y el Dr. David Olvera Pérez, en el turno matutino; en el vespertino, el Dr. Marco Antonio Alarcón, exresidente del mismo servicio, originario de $\mathrm{Ni}$ caragua y nacionalizado mexicano; en la noche, se responsabilizaron los doctores Roberto Blanco Benavides y Gustavo Ramírez Wiella, este último excelente cirujano con formación quirúrgica en Inglaterra, por lo que al servicio se le "inyectaron" otros criterios en cirugía. La demanda de asistencia quirúrgica continuó en ascenso, por lo que pronto pasaron a colaborar en el turno matutino. Rápidamente las cincuenta camas fueron insuficientes, llegando a tener cerca de 200, tomando en cuenta que los pacientes de otros servicios cuando se operaban permanecían en sus camas y no eran trasladados al sexto piso y tampoco al Hospital de Convalecientes. Este servicio permaneció activo hasta el año de 1981, cuando las autoridades del IMSS, apegados a la Declaración de Almá-Atá de 1978, ${ }^{7}$ decidieron reorganizar los servicios asistenciales de salud. Sólo fueron seis años aproximadamente de actividad y productividad del Servicio de Cirugía General del HG del CMN del IMSS.

Bajo la dirección del Dr. Jorge Bautista O'Farrill continuamos con las mismas actividades académicas, sesiones de bibliografía, radiología, anatomía patológica (tejidos), y se incrementaron las cirugías, al implantar la cirugía electiva en el turno vespertino. La producción quirúrgica se mantuvo alrededor de 7,000 cirugías por año, sumando todos los servicios: matutino, vespertino y urgencias.

Siguiendo las disposiciones de la Declaración de Almá-Atá, los servicios de salud fueron divididos en tres niveles de atención: el primer nivel de atención, que es el más cercano a la población, en donde las necesidades de atención se podían resolver con actividades de promoción de salud, prevención de la enfermedad, y otros. En esta puerta de entrada a la salud, se encuentran los establecimientos de baja complejidad, como consultorios generales, centros de salud, policlínicas y otros donde se resuelven aproximadamente el 85\% de los problemas prevalentes. En el segundo nivel de atención, se ubicaron los hospitales y 
establecimientos en donde se impartía atención en las disciplinas de medicina interna, cirugía general, ginecoobstetricia, pediatría y psiquiatría. Se considera que, entre el primer nivel y el segundo nivel, se pueden resolver hasta 95\% de los problemas de salud de la población. En el tercer nivel, se atienden problemas poco prevalentes, patologías complejas que requieren procedimientos especiales y de alta tecnología, se resuelve el $5 \%$ de los problemas que se plantean. Esto explica perfectamente el diseño aplicado en ese tiempo. La jefatura del curso académico de cirugía general se quedó en el HG del CMN del IMSS, pero el servicio asistencial sufrió un cambio irreversible. En el nuevo Hospital de Especialidades del Centro Médico Siglo XXI existían de 20 a 25 camas quirúrgicas para cirugía de "tercer nivel" con cerca de 25 residentes de cirugía general y los médicos de base (que desconozco el número). Ha sido una gran decepción, la relación médico-pacienteproducción asistencial y académica.

El Servicio de Cirugía General pasó a hospitales de segundo nivel, quedando solamente un servicio quirúrgico especial de tercer nivel, cambio trascendente que obligó a los cirujanos aceptar esta nueva disposición, o bien a renunciar. Los cirujanos Bautista, Godínez y Olvera optaron por la segunda opción. Estos cambios abrieron las puertas para que llegaran cirujanos formados en ese hospital, y con una trayectoria quirúrgica notable. El Dr. Andrés Proal fue nombrado como Jefe del Servicio Gastroquirúrgico de tercer nivel junto con dos colaboradores: el Dr. José Luis Ibarrola y el Dr. Luis Sigler. Pero, a partir de esta fecha, ésa es otra historia... que termina en 1985 con el macrosismo.

Otros médicos del Servicio de Gastroenterología que siempre recordaré con mucho afecto por su amistad y sus conocimientos que me trasmitieron son: Luis F. Cervantes $(\dagger)$, Miguel Stoopen, Alfonso Perches, Miguel Tanimoto ( () , Enrique Segovia, José Antonio Vásquez Saavedra, Norberto Treviño García Manzo y Antonio de León.

Finalmente, me gustaría poder nombrar a todos los residentes de cirugía general que pasaron por el HG del CMN del IMSS, pero la lista es grande, y probablemente incurriría en faltas que podrían herir susceptibilidades.
Muchos de ellos son famosos por méritos propios, unos dentro del IMSS, y otros más en otras instituciones. Para muestra, basta señalar que el $30 \%$ de los presidentes de la Asociación Mexicana de Cirugía General fueron residentes del servicio que hoy nos ocupa.

En los próximos capítulos, presentaremos a todos y a cada uno de los médicos y cirujanos que con su labor profesional, de 1963 a 1981, tuvieron una participación importante en la historia que estamos relatando. Su presentación será en orden cronológico, pues al entender los hechos, podremos entender mejor la evolución histórica del servicio de cirugía que llegó a ser.

\section{REFERENCIAS}

1. Guarner V. El significado de la enseñanza de la historia de la medicina en las residencias de cirugía. Gac Méd Méx. 2005; 141: 85-88.

2. Florescano E. La función social de la historia. Presentación para la Cátedra Latinoamericana Julio Cortázar. Guadalajara, Jal; marzo de 2010.

3. Vega V. Diccionario ilustrado de frases célebres y citas literarias. Cicerón. De Oratore, Libro II, Cap. IX, 36. Barcelona: Ed. Gustavo Gill, S.A.; 1955: p. 304.

4. Anzaldo y Regalado V, Nuño-Amezcua E. Gustavo Baz. El Hombre. México, D.F.: Editor Víctor Anzaldo y Regalado: pág. 241.

5. Centro Médico Nacional. Instituto Mexicano del Seguro Social. México: 1963.

6. Hospital General Centro Médico Nacional, IMSS (1963-1985). Editado por el Colegio de Médicos Posgraduados del IMSS. Patrocinio del IMSS. 1994.

7. Declaración de Alma-Atá. Conferencia Internacional sobre Atención Primaria de Salud de Almá-Atá. 6-12 de septiembre 1978.

Consideraciones y responsabilidad ética: Privacidad de los datos. De acuerdo a los protocolos establecidos en el centro de trabajo del autor, este declara que ha seguido los protocolos sobre la privacidad de datos de pacientes preservado su anonimato. El consentimiento informado del paciente referido en el artículo se encuentra en poder del autor.

Financiamiento: No se recibió apoyo financiero para la realización de este trabajo.

Conflicto de intereses: El autor declara que no existe ningún conflicto de intereses en la realización del trabajo.

Correspondencia:

Dr. David Olvera Pérez

E-mail: docolvera2@gmail.com 